\title{
Towards Numerical Simulation Tool of Motion Solid Particles in Fluid Flow
}

\author{
S Zouaoui ${ }^{1 *}$, H Djebouri', B Ferhat ${ }^{2}$, K Mohammedi $^{2}$ \\ 1. LMSE Laboratory, Mechanical Engineering Department, \\ Mouloud Mammeri University of Tizi-Ouzou, Tizi-Ouzou, \\ Algeria \\ 2. Materials, Processes and Environment Research Unit \\ (URMPE), FSI, M'hamed Bougara University of Boumerdes, \\ Algeria.
}

\begin{abstract}
We present here a numerical method to compute the motion of rigid particles in fluid flow with a non-elastic impact law. Many methods have been proposed recently and different strategies have been used to compute such flows. Our motivation is the handling of the non-overlapping constraint in fluid-particle direct simulations. Each particle is treated individually and the Navier-Stokes equations are solved for the moving fluid by Fluent code which is based on the Finite Volume Method. The contacthandling algorithm, which is implemented in a research $\mathrm{C}++$, is based on the projection of the velocity field of the rigid particles over the velocity field of the fluid flow. The method consists of imposing a constraint on the velocity field of the particles, as a guarantee that at each time step the calculated particle velocity field belongs to an eligible velocity field of the fluid. In this case study, an Uzawa algorithm has been applied.
\end{abstract}

\section{INTRODUCTION}

The motion of solid particles in Newtonian and non-Newtonian fluids is not only of fundamental theoretical interest but is also of importance in many industrial applications such as fuel systems for jet engines [1], combustion devices [2], oil cracking [3], slurry flows [4], food processing, building services [5] and pipelines [6]. In all these applications, if the particle density is significant, the interaction between the solid particles and the fluid flow associated to the turbulence effects is not yet well understood.

From a mathematical and numerical standpoint, depending on the length scale at which the fluid-particle flows are described, different approaches are used to describe the behaviour of the two phases. Such problems have motivated the development of numerous algorithms, which can be broadly classified in two broad categories. The first approach is EulerianEulerian, based on a fixed mesh which covers the whole domain where the fluid may be present. Additional constitutive equations may be required to describe interactions between particles [7, 8].

*Corresponding Author: salah.zouaoui@ummto.dz 
The second Methods type is Lagrangian-Eulerian based on a moving mesh, which deforms following the motion of the particle boundaries. To overcome the problems associated with complex geometry by using Cartesian grids throughout the domain, a fictitious domain method has been introduced $[9,10]$ These methods are based on penalty principles [11] It consists on constraining the movement of the fluid to be the same as the movement of a particle by increasing locally the viscosity of the fluid [12-14].

For particle simulation, the discrete element method (DEM) is often used. Models combining the discrete element method for particle modelling and an Eulerian approach to the fluid phase were introduced by Tsuji, Kawaguchi and Tanaka [15]. Since then this approach has been taken up and modified by many researchers [16-21]. This model is implemented on several commercial codes such as ANSYS ${ }^{\circledR}$ Fluent [22] and STAR-CCM+ [23].

The combined computational fluid dynamics-discrete element method (CFD-DEM) is a better and comparatively simpler framework for the simulations of fluid-particles flow [24]. Although the discrete element method allows contact interactions to be taken into account, it has a major disadvantage, which is the need for very small time steps and it is limited by the maximum number of particles that can be simulated [25].

To overcome this problem, in this paper we have proposed a new contact model which is implemented in a research $\mathrm{C}++^{+}$program. This model is semi-implicit in time and is based on non-interpenetration conditions. The strategy adopted is to separate fluid resolution from contact handling. In order to avoid overlapping, the method consists on imposing a constraint on the velocity field of the particle, as a guarantee that at each time step the calculated particle velocity field belongs to an eligible velocity field of the fluid. The numerical procedure is based on the work of Lefebvre and Maury [26, 27].

\section{CONTACT HANDLING PROCEDURE}

Let us detail the method in the case of spherical particles: we denote by $X=\left(x_{i}^{n}\right)_{i=1, N}$ the position of particles (more precisely, the position of their gravity center) at time $t_{n}$, by $\widehat{V}^{n}=\left(\hat{v}_{i}\right)_{i=1, N}$ the a priori translational velocity, by $\widehat{\Omega}^{n}=\left(\widehat{\omega}_{i}\right)_{i=1, N}$ the a priori rotational velocity. As stated before, the a priori updated position of the particles, defined as

$$
X^{n+1}=X^{n}+\Delta t \widehat{V}^{n}+\frac{1}{2} \gamma^{n} \Delta t^{2}
$$

where $\gamma$ is the acceleration, calculated from the Newton's second law. Equation (1) may lead to non-admissible configuration, in the sense that the particles overlap. To avoid this, we project the velocities onto the following set:

$$
K\left(X^{n}\right)=\left\{V \in R^{2 N}, D_{i j}\left(X^{n}\right)+\Delta t G_{i j}\left(X^{n}\right) \cdot V+\frac{1}{2} \gamma^{n} \Delta t^{2} \geq 0, \forall i<j\right\}
$$

where $D_{i j}$ is the distance between every two particles given as (see figure 1):

$$
D_{i j}\left(X^{n}\right)=\left\|x_{i}^{n}-x_{j}^{n}\right\|-\left(R_{i}-R_{j}\right)
$$


We denote by $G_{i j}\left(\boldsymbol{X}^{n}\right) \in R^{2 N}$ the gradient of this distance defined as:

$$
G_{i j}\left(X^{n}\right)=\nabla D_{i j}\left(X^{n}\right)=\left(\ldots, 0,-e_{i j}^{n}, 0, \ldots, 0, e_{i j}^{n}, 0, \ldots\right)
$$

and normal vector is defined as:

$$
e_{i j}^{n}=\frac{x_{i}^{n}-x_{j}^{n}}{\left\|x_{i}^{n}-x_{j}^{n}\right\|}
$$

At each time step, $V \in R^{2 N}$ is an admissible vector if the particles with velocity $V$ do not overlap at the next timestep:

$$
E\left(X^{n}\right)=\left\{V \in R^{2 N}, D_{i j}\left(X^{n}+\Delta t V^{n}+\frac{1}{2} \gamma^{n} \Delta t^{2}\right) \geq 0, \forall i<j\right\}
$$

We not that equation (2) is the linearized form of equation (6) and, furthermore, it can be shown that $K\left(X^{n}\right) \subset E\left(X^{n}\right)$. It means in particular that particles with admissible velocities at time $t_{n}$ do not overlap at time $t_{n+1}$.

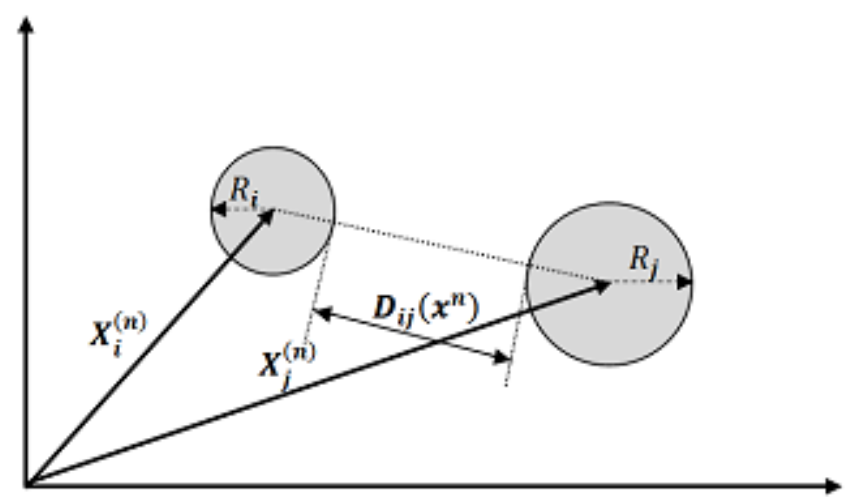

Figure 1: Particles position.

In order to avoid overlapping, the following splitting procedure is proposed: in a first step, we solve the variational problem without taking into account the possible overlapping of the particles (thus defining the a priori velocity of the spheres), then compute the projection of this a priori velocity onto the set of admissible velocities defined by equation (2). The constrained problem is formulated as a saddle-point problem, by using the introduction of Lagrange multipliers:

$$
\left\{\begin{array}{c}
\text { Find }\left(V^{n}, \Lambda^{n}\right) \in \mathbb{R}^{2 N} \times \mathbb{R}_{+}^{N(N-1) / 2} \text { such that } \\
\mathcal{J}\left(V^{n}, \lambda\right) \leq \mathcal{J}\left(V^{n}, \Lambda^{n}\right) \leq \mathcal{J}\left(V, \Lambda^{n}\right), \quad \forall\left(V^{n}, \lambda\right) \in \mathbb{R}^{2 N} \times \mathbb{R}_{+}^{N(N-1) / 2}
\end{array}\right.
$$


with the following functional:

$$
\mathcal{J}(V, \lambda)=\frac{1}{2}\left|V-\hat{V}^{n}\right|^{2}-\sum_{1 \leq i \leq j \leq N} \lambda_{i j}\left(D_{i j}\left(X^{n}\right)+\Delta t G_{i j}\left(X^{n}\right) . V\right)
$$

The number of Lagrange multipliers $\lambda_{i j}$ corresponds to the number of possible contacts. In particular, if there is no contact between particles $i$ and $j$, then $\lambda_{i j}=0$ and the Lagrange multiplier is not activated; conversely, if there is a contact between the two spheres, then $\lambda_{i j}$ may be positive and the corresponding auxiliary field allows the velocity field to satisfy the non-overlapping constraint. The approximate reaction fields $\Lambda^{n}=\Lambda_{i j}^{n}$ is the dual component of a solution to the associated saddle-point problem. The interest in the procedure relies on the possibility to use any suitable solver for the computation of the dynamics. Contacts are handled at a second stage, without any consideration of the proper dynamics. At some point, it allows the use of any solver for the resolution of the dynamics problem: then the so-called predicted velocity field is projected onto the set of admissible velocity fields.

This problem is solved by an Uzawa algorithm [28]. The computation of the admissible translation velocities by projection of the a priori velocities onto the set of admissible translational velocities is done as follows:

$$
V^{n}=\operatorname{argmin}\left(\left|V-\widehat{V}^{n}\right|^{2}, V \in K\left(X^{n}\right)\right)
$$

\subsection{Falling of 50 Particles of Different Sizes on a Plane}

The computer implementation of the contact handling algorithm allows us to simulate the falling of 50 particles of different sizes on a plane (figure 2). This allows us to highlight the particle/particle and particle/wall contact.

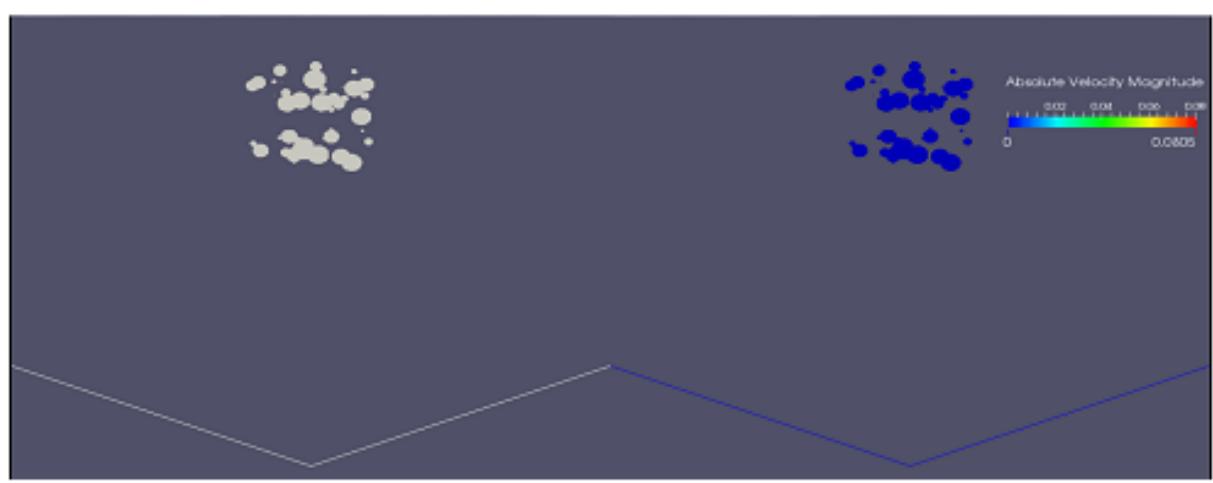

$$
t=0 s
$$




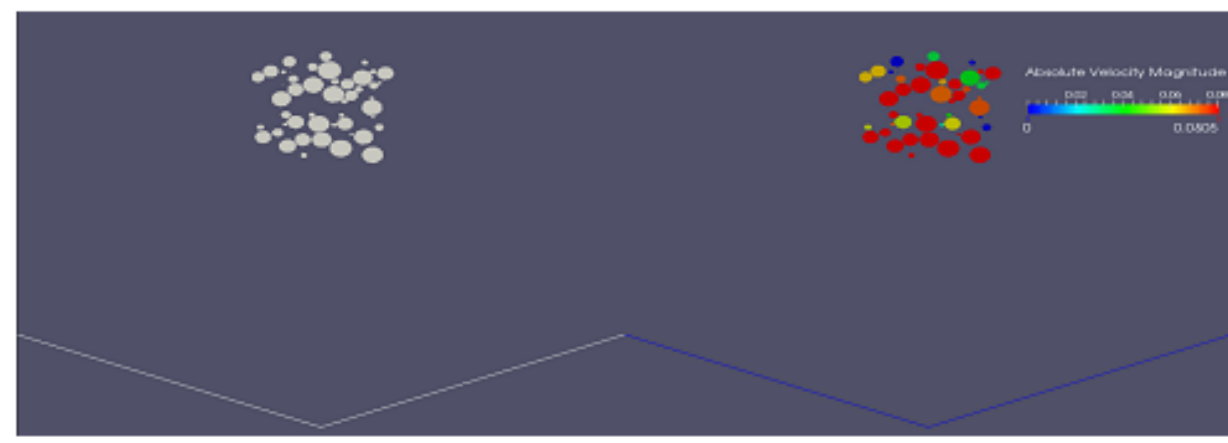

$$
t=1 s
$$

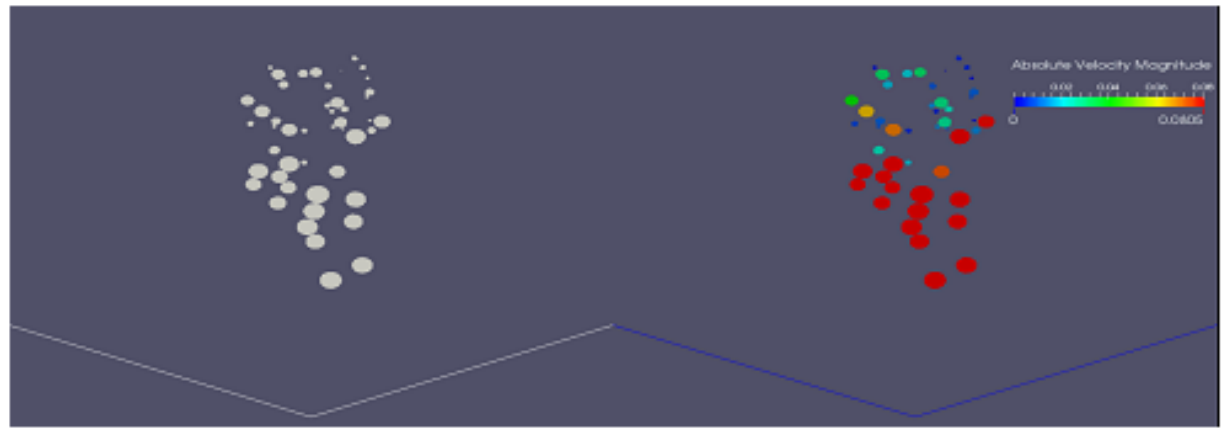

$$
t=10 s
$$

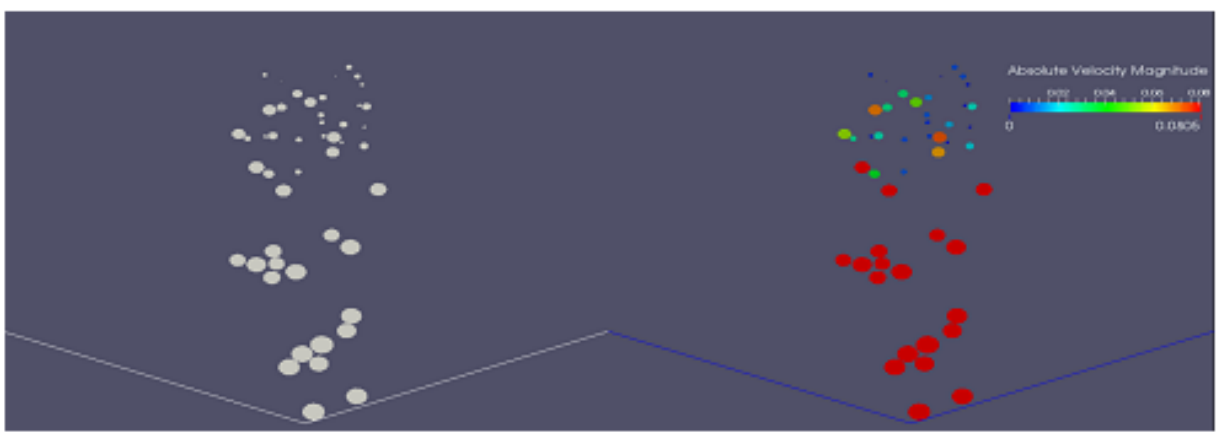

$$
t=20 s
$$




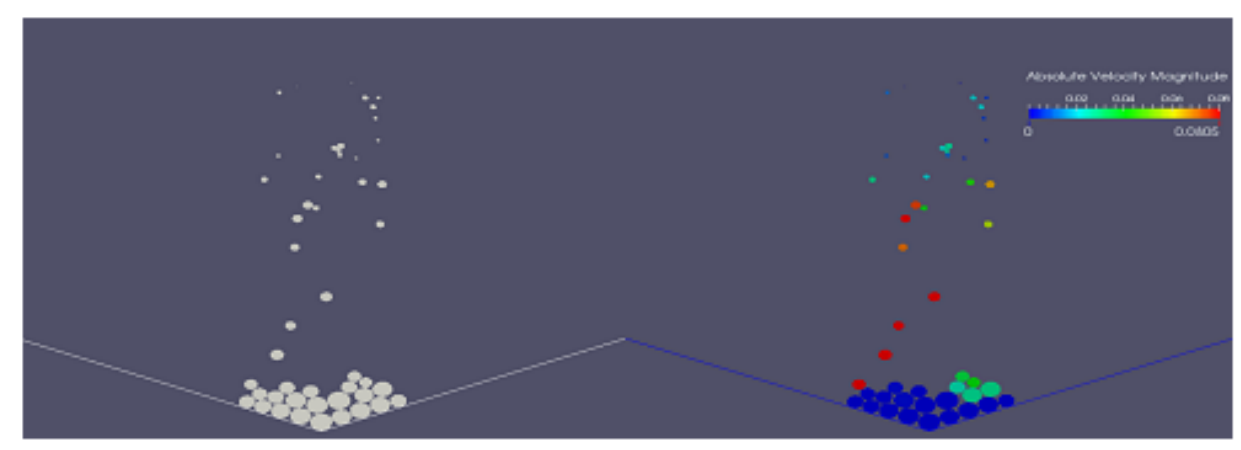

$$
t=60 s
$$

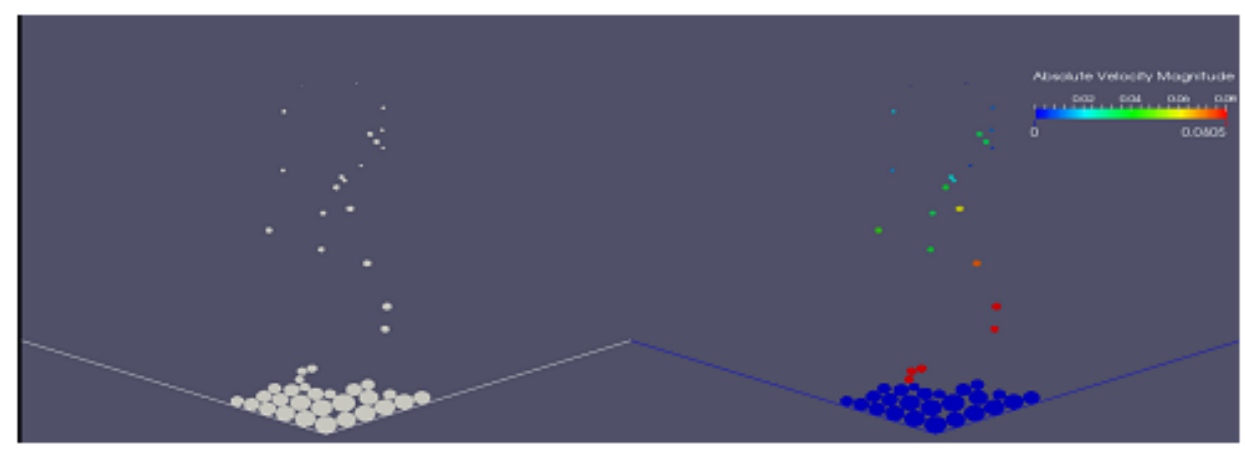

$$
t=80 s
$$

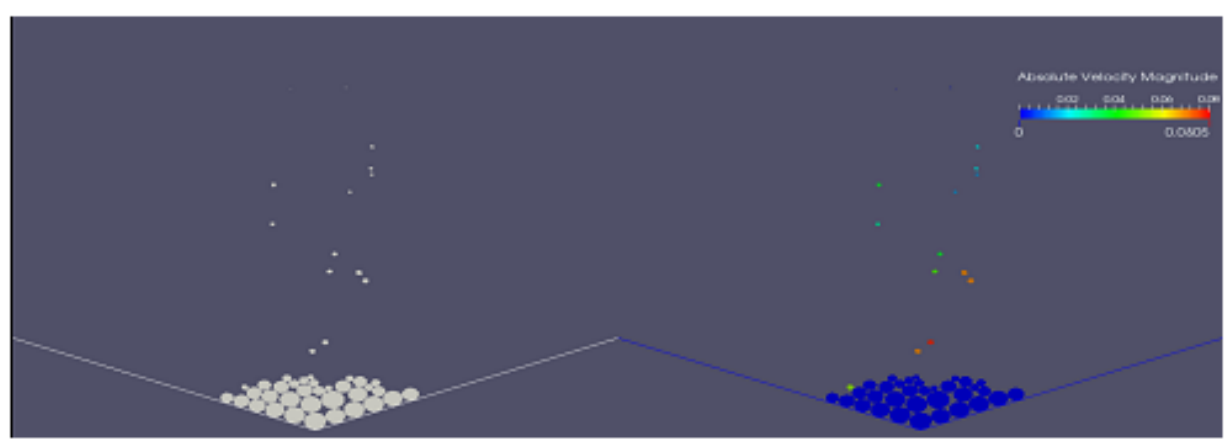

$$
t=100 \mathrm{~s}
$$

Figure 2: Fall down of 50 particles of different sizes on a plane. (left: solid; right: particle velocities) 


\section{FLUID-PARTICLES SIMULATION}

3.1. Simulation of the water flow inside a pipe with obstacles without solid particles

The incompressible Navier-Stokes equations are written in the following form.

$$
\begin{aligned}
\rho_{f}\left(\frac{\partial u}{\partial t}+u . \nabla u\right)-\operatorname{div}(\sigma)=f_{f} & \text { in } \Omega, \\
\operatorname{div}(u)=0 & \text { in } \Omega, \\
u=0 & \text { on } \Omega,
\end{aligned}
$$

where $\rho_{f}$ denotes the density of the fluid, $u\left(u_{1}, u_{2}\right)$ the velocity of fluid, $\sigma$ the stress tensor and $f_{f}=\rho_{f} g e_{y}$ is the external force exerted on the fluid (gravity forces).

We used a Fluent commercial code to solve numerically equation (10). A water flow through a circular pipe of constant cross-section with two singularities is considered (see figure 3). The radius of the pipe is $\mathrm{R}=0.1 \mathrm{~m}$ and its length is $\mathrm{L}=1 \mathrm{~m}$. The boundary conditions are (see figure 4): the parabolic profile of the inlet velocity $V_{z}(y)=V_{\max }\left(1-\frac{y^{2}}{R^{2}}\right)$ with $V_{\max }=2.0 \mathrm{~m} / \mathrm{s}$, and the outlet pressure is equal to the atmospheric pressure, $P_{\text {outlet }}=1 \mathrm{~atm}$. The figures 3 and 5 show respectively the stream lines and the velocity field.

Figure 5 reveals the existence of two recirculation zones after the diaphragm and two others after the second obstacle of triangular shape. On figure 6 it can be seen that the velocity at the wall is zero and reaches its maximum in the throat section. Such a restriction of the passage section is in favour of the increase of the absolute velocity.

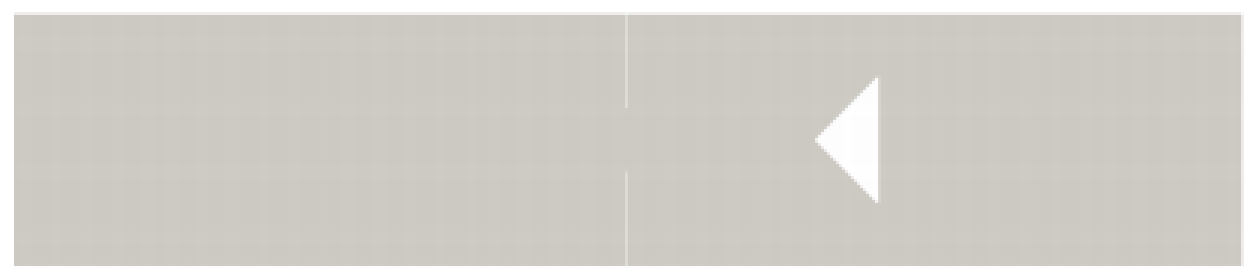

Figure 3: physical domain 


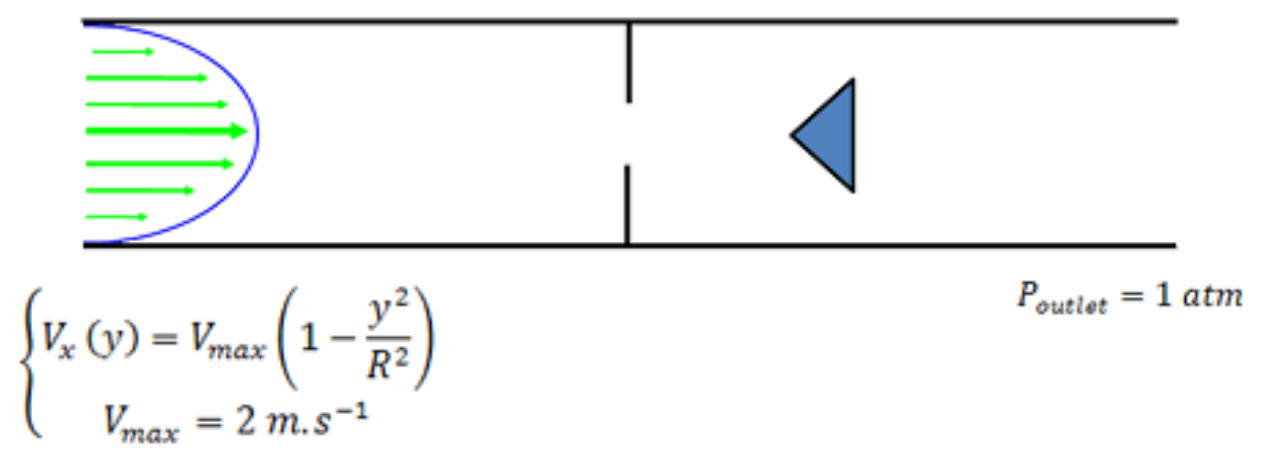

Figure 4: Boundary conditions

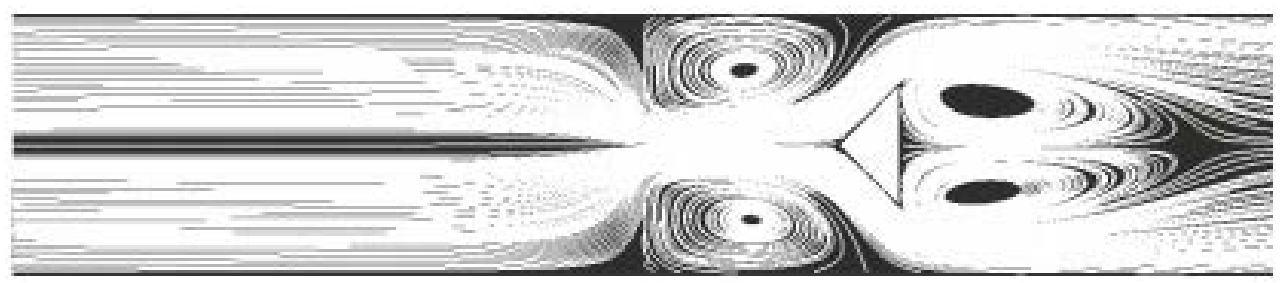

Figure 5: Streamlines

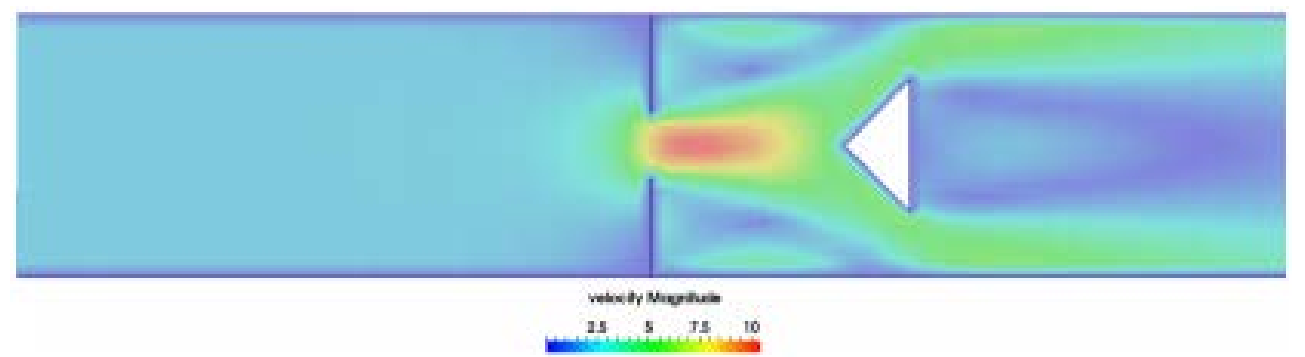

Figure 6: Velocity field

\subsection{Equation of Particles Motion}

The idea here is to consider the solid transport in fluid flow not as the flux of a continuous phase but as the superposition of the motion of individual particles. The motion of each particle is governed by Newton's second law.

$$
m_{p} \frac{d \overrightarrow{U_{p}}}{d t}=\vec{F}_{g}+\vec{F}_{m}+\vec{F}_{\text {Basset }}+\vec{F}_{T}+\vec{F}_{P}+\vec{F}_{g}+\vec{F}_{\text {Magnus }}+\vec{F}_{a f}+\vec{F}_{\text {turb }}
$$

where $m_{p}$ and $\frac{d \overrightarrow{U_{p}}}{d t}$ are the particle mass and acceleration, respectively. The forces acting on the particles are listed in table 1 [29, 30]. 


\begin{tabular}{|c|c|c|}
\hline Force & Designation & Formulation \\
\hline$\vec{F}_{g}$ & Buoyant force & $\left(m_{p}-m_{f}\right) \vec{g}$ \\
\hline$\vec{F}_{m}$ & Added mass force & $m_{f} C_{m} \frac{d\left(\overrightarrow{U_{f}}-d \vec{U}_{p}\right)}{d t}$ \\
\hline$\vec{F}_{\text {Basset }}$ & Basset history force & $\frac{2}{3}\left(d_{p}\right)^{2} \rho_{f} \sqrt{\pi \nu} \int_{-\infty}^{t} \frac{d}{d t}\left(\overrightarrow{u_{f}}-\vec{u}_{p}\right) \frac{d T}{\sqrt{t-T}}$ \\
\hline$\vec{F}_{T}$ & Drag force & $-\frac{1}{2} \rho_{f} \pi R^{2} C_{T}\left|\overrightarrow{U_{p}}-\overrightarrow{U_{f}}\right|\left(\overrightarrow{U_{p}}-\overrightarrow{U_{f}}\right)$ \\
\hline$\vec{F}_{P}$ & Lift force & $-\frac{1}{2} \rho_{f} \pi R^{2} C_{P}\left(\Delta U_{T}^{2}-\Delta U_{B}^{2}\right) \vec{n}$ \\
\hline$\vec{F}_{M a g n u s}$ & Magnus force & $\rho_{p}\left|\overrightarrow{U_{f}}-\overrightarrow{U_{p}}\right|\left(\Omega-\frac{1}{2} \frac{\partial \overrightarrow{u_{f}}}{\partial z}\right) \vec{n}$ \\
\hline$\vec{F}_{a f}$ & Force due to the acceleration of the fluid & $\frac{4}{3} \pi R^{3} \rho_{f} \frac{d \vec{U}_{f}}{d t}$ \\
\hline$\vec{F}_{\text {turb }}$ & Turbulent force & $\frac{1}{2} \rho_{f} \pi R^{2} C_{t u r b}\left|\overrightarrow{U_{f}}\right| U_{y t u r b}$ \\
\hline
\end{tabular}

Table 1: Formulation and designation of the forces acting on the particle

The developed code does not take into account the rotation of the particles, and also neglects the Basset history force. The force due to the acceleration of the fluid is not considered because we are in fully turbulent regime and steady flow for the fluid.

In the literature, there are many correlations for calculating the drag coefficient of particles such as Brown and Lawler's [31, 32] and Flemmer and Banks [33]. In a fully turbulent regime the drag coefficient $C_{T}$ is independent of the particle velocity and Reynolds number [34]. Furthermore, a best and a good approximation equals $C_{T} \approx 0.44$.

According to Auton et al [35], the lift coefficient is taken as $C_{P}=0.5$. Turbulence is implemented by a turbulent force perpendicular to the direction of flow. It is proportional to the product of the flow velocity and the fluctuation of the imposed turbulent velocity, $U_{y t u r b}$ [36].

\subsection{Application: Particles/fluid Flow Simulation inside a Pipe with Obstacles}

By way of application we consider the simple 2D case of water flow inside a pipe with obstacles (see figure 3) in the presence of solid particles.

All particles are initially injected using a Gaussian function at the pipe inlet. The particles diameter ranges from $10^{-2} \mathrm{~m}$ to $30^{-2} \mathrm{~m}$. As soon as the particle is injected, the particle velocity is projected over the flow field velocity space. For this case, 50 particles have been introduced into the flow. Figure 7 shows the evolution of the velocity field of the fluid and the motion of a solid particles over time.

It is clear that the particles are carried by the flow of the fluid. Some particles are trapped in the first obstacle. As it can be seen, most of the particles are close to the bottom wall. Such behaviour is natural since they are solid particles where the effect of weight prevails over other forces and the code takes into account buoyancy force. Nevertheless, the force is not always dominant, which allows some of the particles to follow the fluid flow and not to collide with the wall. This is due to the different size of the particles and the implemented turbulence effect. The existence of certain particles that follow the dynamics of reverse flow can be clearly distinguished. This is a very interesting observation, since it highlights their behaviour in the recirculation zones. 

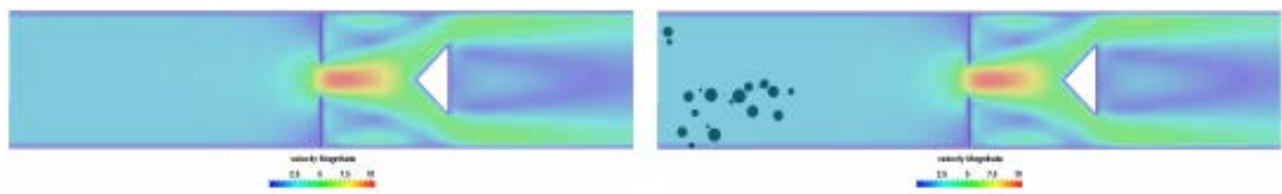

$t=0.0 \mathrm{~s}$

$$
t=0.2 \mathrm{~s}
$$
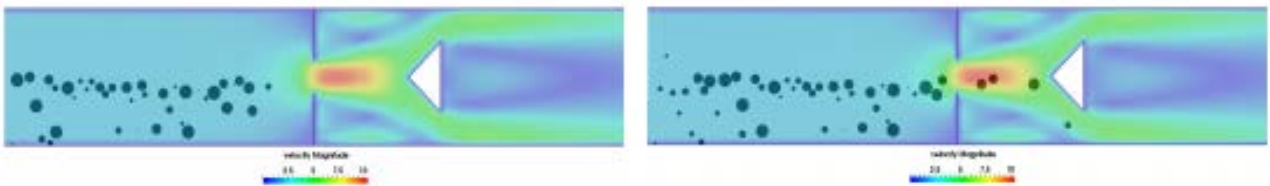

$$
t=0.40 s
$$

$$
t=0.50 \mathrm{~s}
$$
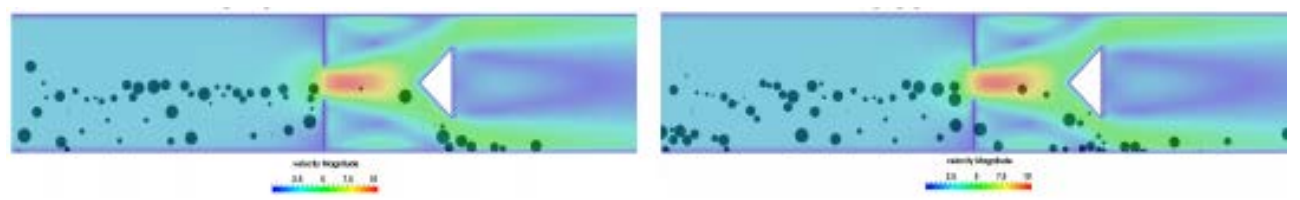

$$
t=0.60 \mathrm{~s}
$$

$$
t=0.80 s
$$
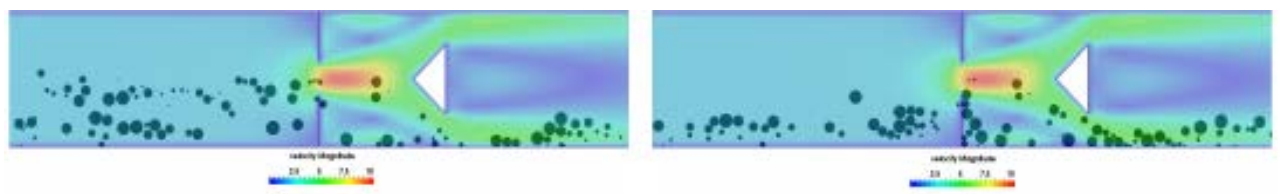

$$
t=1.0 \mathrm{~s}
$$

$$
t=1.50 s
$$
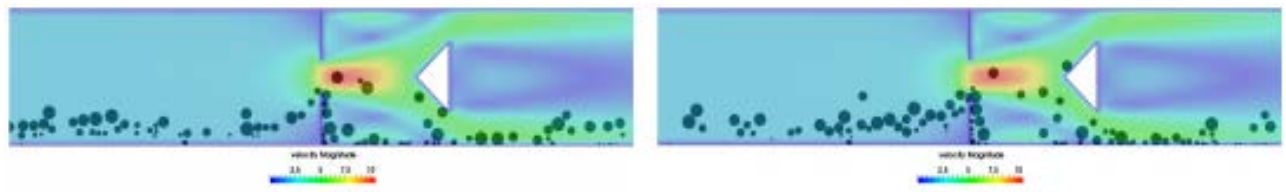

$$
t=2.50 \mathrm{~s}
$$

$$
t=3.0 \mathrm{~s}
$$
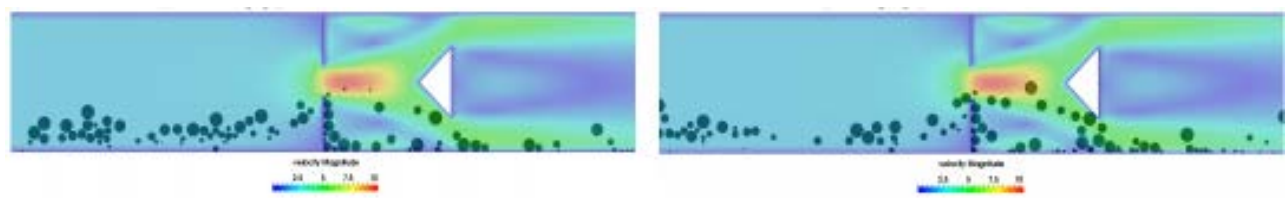

$$
t=3.50 \mathrm{~s}
$$

$$
t=4.0 \mathrm{~s}
$$




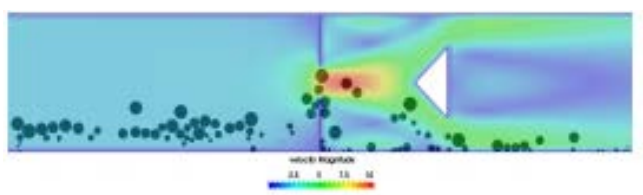

$t=4.50 \mathrm{~s}$

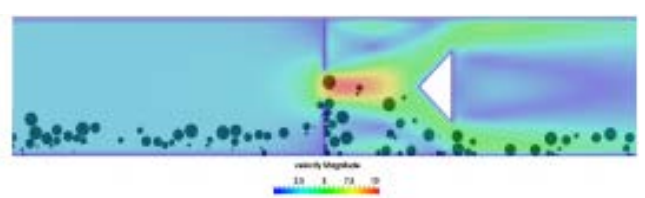

$t=5.0 \mathrm{~s}$

Figure 7: Injection of solid particles: Configuration at different time steps

\section{CONCLUSION AND FUTURE WORK}

Computational fluid mechanics and structure mechanics are two major areas of numerical simulation of physical systems. With the introduction of high performance computing, it has become possible to tackle coupled systems with fluid and structure dynamics. Fluid-particle coupling presents several degrees of complexity. For the study of transport particle in fluid flows, it is recommended to use a Four-way coupling model, where the action of the particles on the fluid is taken into account, as well as inter-particle collisions. Collisions present severe difficulties in direct numerical simulations. In this paper we have proposed a strategy for the numerical modeling of solid transport by using a numerical tool which is efficient in the handling of contacts between particles.

In this work, we simulated the transport of solid particles in a pipe with obstacles. To take into account the solid particles we integrated, in the code of contact handling, the equations of the solid dynamics by considering all the forces acting on a particle in a fluid flow. On the other hand, for the numerical resolution of the Navier-Stokes equations, we resorted to the use of a Fluent commercial code which is based on the finite volume method.

This paper is a contribution to the numerical simulation of solid transport through the implementation of a Contact Handling Procedure. The achieved results also show the importance of the particle's size, since the buoyancy force can be more dominant in certain cases.

As part of this work, a method for simulating the transport of solids in a Newtonian fluid has been developed. This method is based on the Finite element method with penalisation of the tensor of deformation. The fluid behavior is governed by the Navier-Stokes equations within the investigation domain.

Other major improvements regarding the finite volume in-house code, are its extension into three dimensional spaces. 


\section{REFERENCES}

[1] Kuruneru STW, Maréchal E, Deligant M, et al. A Comparative Study of Mixed Resolved-Unresolved CFD-DEM and Unresolved CFD-DEM Methods for the Solution of Particle-Laden Liquid Flows. Archives of Computational Methods in Engineering 2019; 26: 1239-1254.

[2] Minutolo P, Prati MV, Sirignano M, et al. Emission of particles from practical combustion devices burning methane/natural gas. In: European Aerosol Conference. 2009.

[3] Bissell ER, Burnham AK, Braun RL. Shale oil cracking kinetics and diagnostics. Industrial \& Engineering Chemistry Process Design and Development 1985; 24: 381386.

[4] Zouaoui S, Djebouri H, Mohammedi K, et al. Experimental study on the effects of big particles physical characteristics on the hydraulic transport inside a horizontal pipe. Chinese Journal of Chemical Engineering 2016; 24: 317-322.

[5] Müller-Steinhagen H, Malayeri M, Watkinson A. Heat exchanger fouling: environmental impacts. Taylor \& Francis, 2009. Epub ahead of print 2009. DOI: 10.1080/01457630902744119.

[6] Leporini M, Marchetti B, Corvaro F, et al. Sand transport in multiphase flow mixtures in a horizontal pipeline: An experimental investigation. Petroleum 2019; 5: 161-170.

[7] Chen Z, Huan G, Ma Y. Computational methods for multiphase flows in porous media. Siam, 2006.

[8] Liu C, WALkINGTON NJ. An Eulerian description of fluids containing visco-elastic particles. Archive for rational mechanics and analysis 2001; 159: 229-252.

[9] Glowinski R, Pan T-W, Hesla TI, et al. A fictitious domain method with distributed Lagrange multipliers for the numerical simulation of particulate flow. Contemporary mathematics 1998; 218: 121-137.

[10] Glowinski R, Pan T-W, Hesla TI, et al. A fictitious domain approach to the direct numerical simulation of incompressible viscous flow past moving rigid bodies: application to particulate flow. Journal of Computational Physics 2001; 169: 363-426.

[11]Zouaoui S, Djebouri H, Bilek A, et al. Modelling and Simulation of Solid Particle Sedimentation in an Incompressible Newtonian Fluid. Mathematics in Computer Science 2017; $1-13$.

[12] Caltagirone J, Vincent S. Tensorial penalisation method for solving Navier-Stokes equations. COMPTES RENDUS DE L ACADEMIE DES SCIENCES SERIE II FASCICULE B-MECANIQUE 2001; 329: 607-613.

[13] Vincent S, Randrianarivelo TN, Pianet G, et al. Local penalty methods for flows interacting with moving solids at high Reynolds numbers. Computers \& fluids 2007; 36: 902-913.

[14] Vincent S, De Motta JCB, Sarthou A, et al. A Lagrangian VOF tensorial penalty method for the DNS of resolved particle-laden flows. Journal of Computational Physics 2014; 256: 582-614. 
[15] Tsuji Y, Kawaguchi T, Tanaka T. Discrete particle simulation of two-dimensional fluidized bed. Powder technology 1993; 77: 79-87.

[16] Di Renzo A, Di Maio FP. Homogeneous and bubbling fluidization regimes in DEM-CFD simulations: hydrodynamic stability of gas and liquid fluidized beds. Chemical Engineering Science 2007; 62: 116-130.

[17] Hoomans B, Kuipers J, Briels W, et al. Discrete particle simulation of bubble and slug formation in a two-dimensional gas-fluidised bed: a hard-sphere approach. Chemical Engineering Science 1996; 51: 99-118.

[18] Kawaguchi T, Tanaka T, Tsuji Y. Numerical simulation of two-dimensional fluidized beds using the discrete element method (comparison between the two-and threedimensional models). Powder technology 1998; 96: 129-138.

[19] Patankar N, Joseph D. Modeling and numerical simulation of particulate flows by the Eulerian-Lagrangian approach. International Journal of Multiphase Flow 2001; 27: 1659-1684.

[20] Xu B, Yu A. Numerical simulation of the gas-solid flow in a fluidized bed by combining discrete particle method with computational fluid dynamics. Chemical Engineering Science 1997; 52: 2785-2809.

[21] Liu H, Li P, Xiao H, et al. The fluid-solid coupling analysis of screw conveyor in drilling fluid centrifuge based on ANSYS. Petroleum 2015; 1: 251-256.

[22] ANSYS AF. 14.5 User's Guide. ANSYS Inc.

[23] Guide C-AU. STAR-CCM+ Version (7.04). CD-adapco, 2012.

[24] Khawaja H. CFD-DEM simulation of minimum fluidisation velocity in two-phase medium. The International Journal of Multiphysics 2011; 5: 89-100.

[25] Di Renzo A, Cello F, Di Maio FP. Simulation of the layer inversion phenomenon in binary liquid-fluidized beds by DEM-CFD with a drag law for polydisperse systems. Chemical Engineering Science 2011; 66: 2945-2958.

[26] Maury B. A time-stepping scheme for inelastic collisions. Numerische Mathematik 2006; 102: 649-679.

[27] Lefebvre A. Fluid-particle simulations with FreeFem++. In: Esaim: Proceedings. EDP Sciences, 2007, pp. 120-132.

[28] Bank RE, Welfert BD, Yserentant H. A class of iterative methods for solving saddle point problems. Numer Math 1989; 56: 645-666.

[29] Hadji S, Ouahsine A, Naceur H, et al. Modelling of transport and collisions between rigid bodies to simulate the jam formation in urban flows. The International Journal of Multiphysics 2008; 2: 247-266.

[30] Zouaoui S, Aider AA, Djebouri H, et al. Motion of a Solid Particle in a Water Flow Inside a Pipe. In: Exergy for A Better Environment and Improved Sustainability 1. Springer, 2018, pp. 217-231.

[31] Brown PP, Lawler DF. Sphere drag and settling velocity revisited. Journal of Environmental Engineering 2003; 129: 222-231. 
[32] Clift R, Grace JR, Weber ME. Bubbles, drops, and particles. Courier Corporation, 2005. [33] Flemmer R, Banks C. On the drag coefficient of a sphere. Powder Technology 1986; 48: 217-221.

[34] Peker SM, Helvaci SS. Solid-liquid two phase flow. Elsevier, 2011.

[35] Auton T, Hunt J, Prud'Homme M. The force exerted on a body in inviscid unsteady nonuniform rotational flow. Journal of Fluid Mechanics 1988; 197: 241-257.

[36] Tomov P, Khelladi S, Sarraf C, et al. Numerical Modeling of Aerated Cavitation Using a Penalization Approach for Air Bubble Modeling Coupled to Homogeneous Equilibrium Model. In: ASME 2014 International Mechanical Engineering Congress and Exposition. American Society of Mechanical Engineers, 2014, p. V007T09A010-V007T09A010. 